\title{
Relación Entre los Cambios del Ambiente Laboral Ocasionados Postevento Sísmico del 27 Febrero 2010 y la Presencia de Ansiedad y Estrés Laboral en el Profesional de Enfermería
}

\author{
RELATIONSHIP BETWEEN THE CHANGE OF WORK ENVIRONMENT CAUSED BY 27 FEBRUARY 2010 SEISMIC \\ POST-EVENT AND THE PRESENCE OF ANXIETY AND STRESS IN PROFESSIONAL NURSING
}

\author{
Carolina Elena Luengo Martínez'1, Ángela Astudillo Araya'1, Sonia Contreras Contreras' \\ 1. Departamento de Enfermería, UNIVERSIDAD DEL BÍO-BÍO Campus Chillán, Chile.
}

\begin{abstract}
RESUMEN
Objetivo: Determinar la relación entre síntomas de ansiedad y estrés laboral en el profesional de enfermería de dos establecimientos hospitalarios de la Provincia de Nuble con los cambios en el ambiente laboral postevento sísmico. Método: Estudio cuantitativo, correlacional, en 64 profesionales de enfermería. Se aplicó el Maslach Burnout Inventory, la escala de Ansiedad y Depresión de Goldberg y un cuestionario para identificar los cambios en el ambiente laboral postevento sísmico. Resultados: El 56,25\% de los sujetos presentó síntomas de ansiedad, un 59,38\% de depresión, el $84,38 \%$ un nivel medio de estrés. La modificación del espacio físico fue el cambio más estresante. El cambio en la calidad de los insumos utilizados, patología de los usuarios y en el flujograma de atención se asocian estadísticamente con la presencia de ansiedad en los profesionales de enfermería. Conclusión: El evento sísmico ocasionó diversas modificaciones en el ambiente laboral, cuyos cambios pueden ser relacionados con la generación de ansiedad en el profesional de enfermería, que es un pilar fundamental de todas las atenciones de salud; por tanto, en la medida que tengan buena salud mental sus prestaciones serán más efectivas, eficaces y oportunas, incrementando la calidad en la atención a los usuarios.
\end{abstract}

(Luengo C, Astudillo A, Contreras S, 2014. Relación Entre los Cambios del Ambiente Laboral Ocasionados Postevento Sísmico del 27 Febrero 2010 y la Presencia de Ansiedad y Estrés Laboral en el Profesional de Enfermería. Cienc Trab. Ene-Abr; 16 [49]: 1-8).

Palabras clave: SÍNTOMAS DE ANSIEDAD, CAMBIOS EN EL AMBIENTE LABORAL, ESTRÉS LABORAL, EVENTO SÍSMICO, DESASTRE NATURAL.

\section{INTRODUCCION}

La presencia de estrés y ansiedad en el profesional de una institución de salud no siempre es de origen asistencial o por las exigencias del proceso productivo ${ }^{1,2}$ sino por un desajuste entre el trabajador, el puesto de trabajo y la propia organización, exceso de

\section{Correspondencia / Correspondence:}

Carolina Luengo Martínez

Avenida Andrés Bello S/N

Casilla 447 Chillán - Chile

Tel.: 042463089 - 0977048761

e-mail: cluengo@ubiobio.cl; caroluengo@gmail.com

Recibido: 11 de Diciembre 2013 / Aceptado: 08 Enero 2014.

\section{ABSTRACT}

Objective: Determine the relationship between symptoms of anxiety and occupational stress in nursing professionals of two hospitals of the Province of Ñuble with changes in the work environment by seismic post-event. Method: quantitative and correlational study in 64 nurses. The Maslach Burnout Inventory, the Anxiety and Depression Scale of Goldberg and a questionnaire to identify changes in the work environment in seismic post-event, were applied.Results: 56.25\% of the subjects had symptoms of anxiety, 59.38\% of depression, $84.38 \%$ an average stress level. Modification of the physical space was the most stressful change. The change in the quality of supplies used, pathology of user and attention flowchart, associate statistically with the presence of anxiety in nurses.Conclusion: The seismic event caused several changes in the work environment, and those changes can be related to the generation of anxiety in the nursing professional, who is a cornerstone of all health care, therefore, as having good mental health their services will be more effective, efficient and timely, increasing the quality of care to users.

Keywords: ANXIETY SYMPTOMS, CHANGES IN THE WORK ENVIRONMENT, JOB STRESS, SEISMIC EVENT, NATURAL DISASTER. trabajo $^{3,4}$, escasez de recursos físicos, materiales y humanos, entre otros. $^{5}$ Pero sus consecuencias se ven reflejadas en la eficiencia y eficacia de su práctica profesional ${ }^{3,4}$, pudiendo afectar la calidad de la atención. ${ }^{6}$

Es bien conocido que los profesionales dedicados al cuidado o asistencia a los demás, como es el caso del personal sanitario, muestran elevados niveles de estrés, motivado principalmente por las características específicas de su trabajo, que implica el contacto continuo con otras personas, desarrollo de actividades asistenciales, frustración relativa, etc. ${ }^{7}$ Autores como Charoy y Rubio se han planteado que ansiedad y estrés pueden ser considerados sinónimos, por provocar un mismo tipo de reacción emocional, caracterizados por una alta activación fisiológica, siendo la ansiedad una reacción emocional de alerta ante una amenaza, una "respuesta emocional que engloba aspectos cognitivos, fisiológicos y motores". ${ }^{8}$ Y el estrés es un proceso más amplio de adaptación 
al medio. ${ }^{9}$ Se resalta que la ansiedad es uno de los indicadores de estrés que puede volverse patológico a medida que es más intenso. ${ }^{5}$

La ansiedad constituye una reacción normal en la vida de toda persona, a bajos niveles es favorable, ya que motiva y aumenta la capacidad de reacciones. Sin embargo, su exceso o una respuesta exagerada ante estímulos es dañino. Las personas pueden predisponerse a una salud general deficiente. Por otro lado, la ansiedad persistente e intensa a menudo provoca comportamientos nocivos como comer demasiado o consumir alcohol o drogas que se pueden manifestar en forma de crisis. ${ }^{10,11}$

Por otro lado, el término estrés ha sido ampliamente definido, generalmente, refiriéndose a tensión nerviosa, emocional o bien como agente causal de dicha tensión. ${ }^{12}$ El estrés de origen ocupacional se debe a la interacción entre múltiples factores de riesgo e incluye aquellos que provienen del ambiente físico, trastornos de las funciones biológicas, el contenido y organización del trabajo, y diversos componentes psicosociales, tanto laborales como extralaborales. ${ }^{13}$ Entre estos se ha descrito: el contexto macrosocial y el estrato socioeconómico; el tipo de trabajo; el apoyo social, dentro y fuera del trabajo; los sentimientos de autoestima y de control; tipo de personalidad; la edad y el género. ${ }^{14}$

Varios estudios han demostrado que las condiciones en que se desempeña un puesto de trabajo, la oportunidad de controlar, la adecuación entre las exigencias del cargo y las capacidades de la persona que lo desempeña, las relaciones interpersonales, la remuneración y la seguridad física, entre otros, son factores relevantes para el bienestar. ${ }^{13}$ En el nivel psicosomático, la ansiedad, el estrés en diferentes gradaciones se manifiestan por un grupo de sintomas tales como: fatiga, dolores de cabeza frecuentes, trastornos de sueño, pérdida de peso, dolores musculares, entre otros. En el ámbito conductual se hace presente por ausentismo laboral: incapacidad para vivir en forma relajada, superficialidad en el contacto con los demás, comportamientos de alto riesgo. Emocionalmente el individuo comienza a distanciarse como forma de protección del yo, actuando con impaciencia, irritabilidad, incapacidad de concentración ${ }^{10}$, tensión, frustración, ansiedad, depresión y desinterés. $^{7}$

El estrés mantenido y de intensidad elevada en algunos trabajadores -entre los que se encuentran los profesionales de la salud médicos y enfermeros- provoca un sindrome denominado estrés laboral, conocido en inglés como "burnout". ${ }^{10}$

El profesional de enfermería es conocido por estar en alto riesgo de sufrir burnout por la alta carga de trabajo que incluye la interacción estresante y emocional con los demás. A pesar del conocimiento de la relación establecida entre la carga de trabajo y el agotamiento no hay muchos estudios en su impacto. ${ }^{15}$ Un Estudio que se llevó a cabo en enfermeras de 5 países para determinar las causas de ansiedad y estrés describió la "realización de determinadas tareas", "tipo de pacientes" y la "falta de recursos" como fuentes de estrés y ansiedad en el trabajo. ${ }^{16}$

Como se expuso anteriormente, la presencia de estrés y ansiedad en el profesional de una institución de salud no siempre es de origen asistencial; el ambiente laboral es un factor evidentemente relacionado con la generación de ambos, el profesional de enfermería está diariamente enfrentado a fuentes estresoras, siendo los cambios rápidos no compartidos o de las condiciones de trabajo o de responsabilidades algunos de los principales factores generadores de estrés y ansiedad. El verbo cambiar en su expresión más amplia significa "ceder o reemplazar una cosa por otra"; se deno- mina a la "acción de cambiar o a la modificación que resulta de ello". ${ }^{17}$ Este concepto se ha extendido al ámbito de las organizaciones para referirse a la dinámica de estas.

Los eventos de origen telúrico constituyen una realidad frecuente y recurrente en Chile. ${ }^{18}$ Las consecuencias de estos eventos para la población consisten en producción de lesiones o mortalidad, repercusiones psicológicas, pérdidas de tipo material y alteración de los servicios en general. ${ }^{18}$ Las respuestas de las personas a los acontecimientos traumáticos tienen componentes físicos, conductuales y psicológicos ${ }^{19}$ como: los síntomas vinculados a trastornos depresivos y elevados sentimientos de ansiedad ${ }^{19,20}$ son de los más frecuentes en este tipo de fenómenos. ${ }^{21} \mathrm{Y}$ en la atención de salud, suelen ocurrir desde deficiencias transitorias en la oferta sanitaria, hasta grave déficit de atención ocasionado por pérdidas estructurales, no estructurales y de alteraciones organizacionales. ${ }^{18}$

"El sábado 27 de febrero de 2010 Chile se vio sorprendido por un sismo que alcanzó una magnitud de 8,8 en la escala de Richter que tuvo su epicentro frente a Cobquecura. El devastador temblor fue percibido desde la Región de Antofagasta, ubicada al norte del país, hasta la Región de Los Lagos, $2.200 \mathrm{~km}$ al sur, con intensidades de Mercalli que fluctuaron entre los II y los IX grados y afectaron a 13 millones de personas, es decir, aproximadamente al $80 \%$ de la población. El terremoto causó un tsunami que dañó fuertemente las localidades del borde costero de la Región del Maule, en el centro del país, y en la Región del Bío-Bío, al sur, constituyéndose en las zonas más dañadas por esta catástrofe". ${ }^{22}$ Las comunicaciones terrestres y telefónicas se interrumpieron, aislando ciudades y familias. La televisión internacional e internet difundieron la noticia y en pocas horas el desastre se conoció mundialmente. Tal fue la intensidad del sismo que el eje del planeta sufrió un cambio. ${ }^{23}$

"Por otro lado, 135 hospitales (73\% del total del país) presentaron diferentes niveles de daño, 10 de ellos quedaron completamente destruidos y 33, severamente dañados, pero recuperables". ${ }^{23}$ Los servicios de salud se vieron gravemente afectados en toda la zona centro y sur del país debido a la antigüedad de las edificaciones y la magnitud del sismo. Los hospitales de Curicó, Talca, Chillán fueron evacuados exigiendo trasladar a sus pacientes a otros centros hospitalarios o bien atenderlos en hospitales de campaña, lo que ocasionó importantes cambios logísticos-administrativos en el ambiente laboral en que se desempeña el profesional de enfermería como: modificaciones de servicio, de turnos, cambios de jefatura, modificación de horarios, cambios del equipo de trabajo, entre otros. Todo esto para hacer frente a las demandas generadas por la catástrofe. Un desastre produce un cambio o modificación en la capacidad para llevar a cabo algunas de las actividades diarias, provocando una interrupción en los hábitos, roles y rutinas afectando negativamente a la adaptación ocupacional y provocando vulnerabilidad tanto individual como colectiva, ${ }^{24}$ generando desorganizaciones personales y sociales, entre otras ${ }^{25}$ y pudiéndose convertir en una fuente importante de estrés y ansiedad. Cuando acontecimientos trágicos ocurren, la capacidad habitual de alguien de adaptarse puede ser interrumpida. ${ }^{26}$ Los desastres naturales, entendidos como cualquier emergencia que sobrepase las capacidades de respuesta local, obligando a solicitar apoyo externo ${ }^{27}$, actúan como agente estresor, sometiendo al aparato psíquico a un conflicto, donde la persona pondrá en juego distintas reacciones que darán lugar a un proceso cognitivo comportamental. Este proceso permitirá que el individuo, en función de sus recursos (materiales, intelectuales, físicos, psíquicos y emocionales), pueda 
tener una respuesta más o menos adaptada. Sin embargo, si las capacidades de dar respuesta al agente estresor se ven sobrepasadas por el evento de excepción, lo más probable es que la persona padezca de elevados niveles de estrés. ${ }^{28}$ La OPS también afirma que la catástrofe en sí como todos sus efectos colaterales generan frecuentemente trastornos de ansiedad y sintomas depresivos o ansiosos ${ }^{21}$, dentro de los cuales se encuentra el trastorno por estrés postraumático (TEPT) y la crisis de angustia o ataque de pánico (CAPA); los trastornos del ánimo como la depresión; los trastornos orgánicos de causa psíquica; el consumo excesivo de alcohol y drogas; y las conductas violentas, como la violencia intrafamiliar. $^{21}$

Se sabe que al igual que en otros eventos traumáticos (p. e., guerra, secuestros, huracanes) las personas frente a este tipo de fenómenos naturales son expuestas a situaciones que vulneran sus capacidades, alterando su salud mental tanto a corto como a largo plazo. ${ }^{21}$ Inmediatamente después del desastre, respuestas emocionales en aquellos directamente afectados por el desastre pueden incluir una ansiedad difusa y en particular una pérdida de sentido de la seguridad. ${ }^{26}$

En esta perspectiva, diversas investigaciones plantean que frente a la imposibilidad de responder o adaptarse rápidamente a un evento significativo e inesperado, como el terremoto recién pasado, y sus múltiples réplicas procedidas a diversa intensidad, es probable que una persona continúe sintiéndose afectada durante varios meses con posterioridad al evento, e inclusive manifieste signos de estrés, que se expresarían con diferente sintomatología, y en diversos ámbitos tales como el familiar, social y laboral ${ }^{28}$, los cuales emergen con mayor intensidad varios meses posterior al evento, requiriéndose una atención prolongada, sobre todo cuando se requiere reconstruir hábitats y retomar el curso de la vida. ${ }^{25}$

Algunos de estos desórdenes mejoran con el paso del tiempo, mientras que otros se mantienen incluso hasta años después de la catástrofe. ${ }^{21}$ Aunque hoy se sabe que la mayoria de los afectados por un desastre no desarrollará psico-patología, un grupo significativo lo hará, destacándose estrés, depresión y ansiedad. ${ }^{27}$

No obstante, según lo señalado por Cohen (2008), es preciso tener en cuenta que la presencia de este tipo de síntomas no implica necesariamente la existencia de una patología y que, al observar y documentar síntomas después de un desastre, estos deberían interpretarse como procesos y manifestaciones para adaptarse al estrés post-desastre, más que como enfermedades. ${ }^{21}$

Desde la perspectiva ocupacional pueden modificar el desempeño ocupacional de forma permanente o temporal, ya que tienen repercusiones en el contexto y en el entorno cotidiano de la persona y los patrones de desempeño pueden llegar a interrumpirse por la pérdida de la actividad productiva. ${ }^{24}$

Dado que las personas necesitan generar recursos económicos para subsistir, el ámbito laboral es ineludible. Ante la imposibilidad de adaptarse gradualmente al trabajo, sumado a la sensación de falta de control, estaría limitada la capacidad de generar alguna estrategia planificada de adaptación, por lo que el estrés que percibe la persona debería incrementarse, llegando a influir en la manera de interpretar la realidad. Otros problemas relacionados con el estrés pueden afectar otras dimensiones tales como la satisfacción laboral y el compromiso organizacional. ${ }^{28}$

Las autoridades nacionales y regionales han avanzado mucho en rehabilitar la infraestructura perdida; se mantendrá una alteración en la atención que se recuperará completamente al menos cuatro años después de la ocurrencia del terremoto. ${ }^{22}$
A pesar del gran número de investigaciones que evalúan el impacto en la salud mental de las personas después de un desastre natural, la investigación relacionada con los efectos de una catástrofe natural en los recintos hospitalarios y los cambios del ambiente laboral que esto desencadena es muy escasa, por no decir inexistente y más aun el impacto que esto conlleva en la salud mental de los trabajadores que ahí se desempeñan, como es el caso del profesional de enfermería. De aquí se desprende la relevancia de este estudio, ya que el personal en cuestión es la fuerza de trabajo más representativa de las actividades médicas de los centros de atención de salud. ${ }^{30}$ Por lo tanto, es esencial que los cuidados de enfermería al cliente con problemas de salud que afectan seriamente su calidad de vida sean administrados por personal de enfermería motivado, capaz de individualizar las necesidades de cada cliente sin lesionar su estabilidad psicológica. Los resultados de este estudio permitirán conocer los cambios del ambiente laboral con mayor repercusión en la salud mental de los profesionales de enfermería y por un lado poder desarrollar programas de intervención. Distintas investigaciones internacionales evidencian la necesidad de instaurar programas de prevención/intervención para los profesionales con estrés y/o ansiedad. ${ }^{31}$ Por otro lado, saber afrontar de mejor modo situaciones de igual índole, ya que el destino de la organización depende en gran parte de sus recursos humanos, si estos son capaces pueden enfrentar con éxito la competencia y aprovechar al máximo los recursos técnicos y materiales de que se dispone: entonces la organización podrá lograr sus objetivos.

Por tanto, el objetivo del presente estudio pretende determinar la relación entre sintomas de ansiedad y estrés laboral en el profesional de enfermería de establecimientos hospitalarios de la provincia de Ñuble con los cambios en el ambiente laboral generados postevento sísmico.

\section{MATERIAL Y MÉTODOS}

Estudio de carácter cuantitativo, correlacional, de corte transversal. Se estudiaron 64 profesionales de enfermería con cargo de "Enfermera/o Clínica/o", de los servicios de Medicina, Cirugía, Urgencia y Pediatría, pertenecientes a dos hospitales de la Provincia de Nuble, los cuales sufrieron graves daños estructurales que obligaron a realizar una serie de cambios logístico-administrativos. Se excluyeron a enfermeros que se encontraban con licencia médica, vacaciones, días administrativos o por no estar en el servicio durante el periodo de recolección de datos; se excluyeron además a quienes negaron dar su consentimiento, aquellos que se encontraban realizando reemplazos. Se estudiaron variables sociodemográficas, laborales, de salud y los cambios en el ambiente laboral, donde se consideró: cambios de espacio físico (servicio, establecimiento), jefatura/línea de dependencia, equipo de trabajo, dotación de personal, organizacionales (turnos, rutina diaria, flujograma de atención), usuario a atender (edad, patología, complejidad), insumos-equipamiento. Se elaboró por los investigadores un protocolo de acción o de campo para obtener resultados coherentes entre sí, con bajos niveles de discordancia, con el fin de evitar sesgos en el proceso de recolección de datos, los cuales se obtuvieron a través de la autoaplicación del Maslach Burnout Inventory, creado por Maslash y Jackson en 1981, adaptado y validado por Gil Monte y Peiró en el año 1999, España. A nivel nacional la validación se realizó mediante la investigación de 
Jélvez, Ibañes y Olivares "Validez factorial del Maslash Burnout Inventory Human Services (MBI-HSS) en profesionales chilenos". ${ }^{32}$ El cuestionario consta de 22 items que se distribuyen en tres escalas denominadas: agotamiento emocional (9 ítems), realización personal en el trabajo (8 items), y despersonalización (5 items). Se empleó la escala de tipo-Likert, indicando la recurrencia con la que han experimentado la situación descrita en cada uno de ellos, cuya escala tiene 7 grados que van de 0 ("nunca") a 6 ("todos los días"); la escala de Ansiedad y Depresión de Goldberg: este cuestionario fue desarrollado en 1988 a partir de una versión modificada de la Psychiatric Assessment Schedule, con la finalidad de lograr una entrevista de corta duración para ser utilizada por médicos no psiquiatras como instrumento de cribaje. La versión en castellano ha sido validada por Montón C. y cols. (1993). La versión castellana ha demostrado su fiabilidad y validez en el ámbito de la atención primaria y tiene una sensibilidad (83,1\%), especificidad (81,8\%) y valor predictivo positivo $(95,3 \%)$ adecuados. ${ }^{33}$ Consta de dos escalas, una de ansiedad y otra de depresión, con 9 ítems cada una, todos ellos de respuesta dicotómica (sí/no); se da una puntuación independiente para cada escala, con un punto para cada respuesta afirmativa y un cuestionario para identificar los cambios logísticos y administrativos en el ambiente laboral postevento sísmico elaborado por las autoras, validado por prueba piloto y revisión de expertos, el cual consideró las variables expuestas anteriormente. Para el análisis estadístico se utilizaron medidas de tendencia central. Para probar diferencia de proporciones se aplicó prueba de $\mathrm{Z}$ score. El análisis inferencial o bivariado estuvo compuesto por la prueba de asociación chi cuadrado, más el cálculo de Odds-Ratio con 95\% de confianza. Se solicitó mediante carta adjunta el consentimiento de dichos profesionales, conservando anonimato y confidencialidad, respetando la libertad del individuo en cualquier momento del estudio. Previo a lo anterior se solicitó autorización para la aplicación de los instrumentos de investigación a los directores de los hospitales en estudio y a la subdirectora de enfermería o enfermera coordinadora, según el caso de cada establecimiento.

\section{RESULTADOS}

Se estudiaron a 64 profesionales de enfermería de 4 servicios clínicos de dos hospitales de la Provincia de Nuble, Región del Bío-Bío, ambos establecimientos vinculados a la red asistencial pública de salud del Ministerio de Salud, Chile.

\section{Análisis Exploratorio de Datos}

La edad media de los sujetos estudiados es de 37 años, entre 10 a 12 años de servicio y 10 años de antigüedad en el servicio clínico. El 85,94\% son mujeres y el 67,19\% indicaron no presentar una pareja actual $(\mathrm{p}<0,001)$.

El 85\% de los profesionales de enfermería señaló no presentar enfermedades crónicas no transmisibles previamente diagnosticadas ( $\mathrm{p}<0,001)$. El 82,21\% señaló no haber presentado licencia médica, de lo que sí presentó fue de tipo psiquiátrica, anatómicofuncional y tipo orgánica.

El 56,25 \% presentó síntomas de ansiedad y 59,38\% (p < 0,05) de depresión; un 84,38\% presentó un nivel medio de estrés y un $12,50 \%$, alto ( $\mathrm{p}<0,001)$.

En relación a la proporción de profesionales de enfermería que ha reportado cambios que se sucedieron con motivo de la reestructuración logística, técnica- administrativa sufrida con ocasión del sismo, se observa que se presentaron cambios estadísticamente no significativos de servicio clínico $(22,58 \% ; p=1,000)$, de establecimiento $(14,75 \% ; p=1,000)$, de jefe $(33,33 \% ; p=$ $0,999)$, de turno $(13,11 \% ; \mathrm{p}=1,000)$, de equipo de trabajo $(45,16 \% ; p=0,8633)$; de complejidad respecto al usuario a atender $(53,13 \% ; p=0,2398)$, de rutina de trabajo $(42,86 \% ; p=$ 0,9469), de cantidad de insumos necesarios para atender a los usuarios $(45,31 \% ; p=0,8557)$, de equipos e insumos $(44,44 \% ; p$ $=0,8958)$, de dotación del personal de enfermería $(39,06 \% ; \mathrm{p}=$ 0,9933), en el tipo de usuario a atender $(23,81 \%$; $p=1,000)$, de tipo de patología $(34,38 \% ; p=0,9998)$ y cambio en el flujograma de trabajo $(44,26 \% ; p=0,9030)$.

Los cambios observados por los profesionales de enfermería que presentaron una proporción estadísticamente significativa corresponden a modificación del espacio de trabajo $(78,13 ; \mathrm{p}<0,001)$ y calidad de los insumos para la atención del usuario $(57,81 \% ; \mathrm{p}=$ 0,0386).

Respecto a la cantidad de cambios, el 40,63\% de los profesionales de enfermería estudiados presentó menos de 5 cambios, el 42,19\% entre 5 y 9 y el 17,19\% entre 10 y 14 cambios en comparación al estado anterior al sismo y en referencia de la percepción de los profesionales de enfermería respecto a los cambios que se presentaron luego del sismo, 12,7\% ( $\mathrm{p}=1,000)$ percibió que los cambios le favorecieron, y 40\% ( $\mathrm{p}=0,9882)$, indicó que le perjudicó; por otro lado, el 47,83\% ( $\mathrm{p}<0,001$ ) indicó que el cambio que mayor estrés le produjo fue la modificación en el espacio físico donde trabaja.

\section{Análisis bivariado}

Se identifican los factores asociados con sintomas de ansiedad producidos en profesionales de enfermería expuestos a cambios en su ambiente laboral luego del sismo de 27 de febrero de 2010 de 8,8 grados Richter.

\section{Tabla 1.}

Sintomas de ansiedad según caracteristicas generales de los profesionales de enfermería en estudio $(n=64)$.

\begin{tabular}{|c|c|c|c|c|}
\hline Caracteristicas & Síntoma & Ansiedad & OR & $p$ \\
\hline Sexo & $\begin{array}{c}5(13,9) \\
31(86,1)\end{array}$ & $\begin{array}{l}4(14,3) \\
24(85,7)\end{array}$ & $\begin{array}{c}0,968 \\
{[0,234-3,998]}\end{array}$ & 0,964 \\
\hline $\begin{array}{l}\text { Edad } \\
\qquad(20-44 / 45-60)\end{array}$ & $\begin{array}{c}27(75,0) \\
9(25,0)\end{array}$ & $\begin{array}{l}20(71,4) \\
8(28,6)\end{array}$ & $\begin{array}{c}1,200 \\
{[0,394-3,656]}\end{array}$ & 0,784 \\
\hline $\begin{array}{l}\text { Pareja } \\
\text { (si/no) }\end{array}$ & $\begin{array}{l}25(69,4) \\
18(64,3)\end{array}$ & $\begin{array}{l}11(30,6) \\
10(35,7)\end{array}$ & $\begin{array}{c}1,263 \\
{[0,442-3605]}\end{array}$ & 0,663 \\
\hline $\begin{array}{l}\text { ECNT } \\
\text { (si/no) }\end{array}$ & $\begin{array}{c}5(15,4) \\
28(84,8)\end{array}$ & $\begin{array}{c}4(14,8) \\
23(85,2)\end{array}$ & $\begin{array}{c}1,012 \\
{[0,247-4,273]}\end{array}$ & 0,971 \\
\hline $\begin{array}{l}\text { Antigüedad } \\
\qquad(1-20 / 21-39)\end{array}$ & $\begin{array}{c}32(88,9) \\
4(11,1)\end{array}$ & $\begin{array}{c}21(75,0) \\
7(25,0)\end{array}$ & $\begin{array}{c}2,667 \\
{[0,694-10,247]}\end{array}$ & 0,144 \\
\hline $\begin{array}{l}\text { Años de servicio } \\
\quad(1-20 / 21-39)\end{array}$ & $\begin{array}{c}30(83,3) \\
6(16,7)\end{array}$ & $\begin{array}{l}19(67,9) \\
9(32,1)\end{array}$ & $\begin{array}{c}2,36 \\
{[0,726-7,724]}\end{array}$ & 0,147 \\
\hline $\begin{array}{l}\text { Licencia } \\
\text { (si/no) }\end{array}$ & $\begin{array}{c}9(25,0) \\
27(75,0)\end{array}$ & $\begin{array}{c}2(7,1) \\
26(92,9)\end{array}$ & $\begin{array}{c}4,333 \\
{[0,854-21,986]}\end{array}$ & 0,060 \\
\hline
\end{tabular}

OR=Odds Ratios. IC 95\% Intervalo de confianza al 95\%.

Prueba de asociación de ${ }_{-}$.

La Tabla $\mathrm{N}^{\circ} 1$ indica que las variables sociodemográficas, laborales y de salud estudiadas son independientes de sintomas ansiosos (todos con valores $\mathrm{p}>0,05$ ). 
Tabla 2.

Síntomas de ansiedad según cambios presentados en su ambiente laboral con ocasión del sismo 8,8 $(n=64)$.

\begin{tabular}{|c|c|c|c|c|}
\hline $\begin{array}{l}\text { Cambios Post } \\
\text { Sismo en: }\end{array}$ & \multicolumn{2}{|c|}{ Síntomas de Ansiedad } & $\begin{array}{l}\text { OR } \\
\text { IC } 95 \%\end{array}$ & $p$ \\
\hline \multirow{2}{*}{$\begin{array}{l}\text { Servicio } \\
\text { (si/no) }\end{array}$} & $10(28,6)$ & $4(14,8)$ & 2,300 & \\
\hline & $25(71,4)$ & $23(85,2)$ & {$[0,633-8,359]$} & 0,199 \\
\hline \multirow{2}{*}{$\begin{array}{l}\text { Establecimiento } \\
\quad(\mathrm{s} / \mathrm{no})\end{array}$} & $5(14,7)$ & $4(14,8)$ & 0,991 & \\
\hline & $29(85,3)$ & $23(85,2)$ & {$[0,239-4,118]$} & 0,990 \\
\hline \multirow{2}{*}{$\begin{array}{l}\text { Jefatura } \\
\text { (si/no) }\end{array}$} & $12(34,3)$ & $9(32,1)$ & 1,101 & \\
\hline & $23(65,7)$ & $19(67,9)$ & {$[0,383-3,168]$} & 0,858 \\
\hline \multirow{2}{*}{$\begin{array}{l}\text { Turno } \\
\text { (si/no) }\end{array}$} & $7(20,6)$ & $1(3,7)$ & 6,741 & \\
\hline & $27(79,4)$ & $26(96,3)$ & {$[0,775-58,648]$} & 0,052 \\
\hline \multirow{2}{*}{$\begin{array}{l}\text { Equipo de trabajo } \\
\text { (si/no) }\end{array}$} & $18(51,4)$ & $10(37,0)$ & 1,800 & \\
\hline & $17(48,6)$ & $17(63,0)$ & {$[0,46-5,014]$} & 0,259 \\
\hline \multirow{2}{*}{$\begin{array}{l}\text { Modificación del espacio } \\
\text { (si/no) }\end{array}$} & $30(83,3)$ & $20(71,4)$ & 2,000 & \\
\hline & $6(16,7)$ & $8(28,6)$ & {$[0,602-6,642]$} & 0,253 \\
\hline \multirow{2}{*}{$\begin{array}{l}\text { Complejidad del usuario } \\
\text { (si/no) }\end{array}$} & $23(63,9)$ & $11(39,3)$ & 2,734 & \\
\hline & $13(36,1)$ & $17(60,7)$ & {$[0,987-7,573]$} & 0,050 \\
\hline \multirow{2}{*}{$\begin{array}{l}\text { Rutina de trabajo } \\
\text { (si/no) }\end{array}$} & $17(47,2)$ & $10(37,0)$ & 1,521 & \\
\hline & $19(52,8)$ & $17(63,0)$ & {$[0,549-4,214]$} & 0,419 \\
\hline \multirow{2}{*}{$\begin{array}{l}\text { Calidad de insumos } \\
\text { (si/no) }\end{array}$} & $26(72,2)$ & $11(39,3)$ & 4,018 & \\
\hline & $10(27,8)$ & $17(60,7)$ & {$[1,403-11,508]$} & 0,008 \\
\hline \multirow{2}{*}{$\begin{array}{l}\text { Cantidad de insumos } \\
\text { (si/no) }\end{array}$} & $19(52,8)$ & $10(35,7)$ & 2,012 & \\
\hline & $17(47,2)$ & $18(64,3)$ & {$[0,731-5,539]$} & 0,174 \\
\hline \multirow{2}{*}{$\begin{array}{l}\text { Equipos/instrumentos } \\
\text { (si/no) }\end{array}$} & $16(45,7)$ & $12(42,9)$ & 1,123 & \\
\hline & $19(54,3)$ & $16(57,1)$ & {$[0,413-3,056]$} & 0,821 \\
\hline \multirow{2}{*}{$\begin{array}{l}\text { Personal de enfermería } \\
\text { (si/no) }\end{array}$} & $17(47,2)$ & $8(28,6)$ & 2,237 & \\
\hline & $19(52,8)$ & $20(71,4)$ & {$[0,784-6,386]$} & 0,129 \\
\hline \multirow{2}{*}{$\begin{array}{l}\text { Usuario a atender } \\
\text { (si/no) }\end{array}$} & $10(28,6)$ & $5(17,9)$ & 1,840 & \\
\hline & $25(71,4)$ & $23(82,1)$ & {$[0,547-6,193]$} & 0,321 \\
\hline \multirow{2}{*}{$\begin{array}{l}\text { Patología de usuarios } \\
\text { (si/no) }\end{array}$} & $17(47,2)$ & $5(17,9)$ & 4,116 & \\
\hline & $19(52,8)$ & $23(82,1)$ & {$[1,280-13,230]$} & 0,014 \\
\hline \multirow{2}{*}{$\begin{array}{l}\text { Flujograma de atención } \\
\text { (si/no) }\end{array}$} & $22(61,1)$ & $5(20,0)$ & 6,286 & \\
\hline & $14(38,9)$ & $20(80,0)$ & {$[1,918-20,603]$} & 0,001 \\
\hline
\end{tabular}

OR=Odds Ratios. IC 95\% Intervalo de confianza al 95\%.

Prueba de asociación de _2 2 .

En esta tabla se destaca que el cambio en la calidad de los insumos utilizados ( $O R=4,018)$, patología de los usuarios $(O R=4,116)$ y en el flujograma de atención $(\mathrm{OR}=6,286)$, se asocian estadísticamente con la presencia de ansiedad en los profesionales de enfermería (todos con valores $\mathrm{p}<0,05$ ).

En relación a la cantidad de cambios, los profesionales de enfermería que contabilizaron entre 0 y 4 cambios con ocasión del sismo, se asocian significativamente con síntomas de ansiedad ( $O R=0,288 ; p$ $=0,017)$; no así quienes reportaron entre 5 y 9 cambios $(\mathrm{OR}=1,179$; $\mathrm{p}=0,749)$ y entre 10 y 14 cambios $(\mathrm{OR}=0,920 ; \mathrm{p}=0,900)$. Considerando los valores OR, no se observa un gradiente de asociación entre el número de cambios y los síntomas de ansiedad de los profesionales de enfermería en estudio. Los cambios que le han favorecido y perjudicado en su trabajo son independientes de los sintomas de ansiedad (ambos con $\mathrm{p}>0,05$ ).

El estrés laboral se asocia significativamente con sintomas de ansiedad ( $\mathrm{p}<0,05)$; es así que la presencia de estrés aumenta en 4,68 veces el riesgo de presentar síntomas de ansiedad en los profesionales en estudio (IC 95\% 1,67-13,546). Dado que la variable estrés laboral se encuentra asociada con ansiedad laboral y pueda modificar el efecto con la asociación de los cambios ocurridos con ocasión del sismo, se considerará como posible factor de confusión en la próxima tabla.
Tabla 3.

Sintomas de ansiedad de los profesionales de enfermeria en estudio según cambios presentados en su ambiente laboral con ocasión del sismo $8,8^{\circ}$ considerando el posible efecto del estrés laboral $(n=64)$.

\begin{tabular}{|c|c|c|c|c|}
\hline $\begin{array}{l}\text { Cambios Post } \\
\text { Sismo en: }\end{array}$ & $\begin{array}{l}\text { Sintomas } \\
\text { Si presenta }\end{array}$ & $\begin{array}{l}\text { e Ansiedad } \\
\text { No presenta }\end{array}$ & $\begin{array}{c}\text { OR } \\
\text { IC } 95 \%\end{array}$ & $p$ \\
\hline \multirow{2}{*}{$\begin{array}{l}\text { Calidad de insumos } \\
\text { (si/no) }\end{array}$} & $26(72,2)$ & $11(39,3)$ & 4,018 * & \\
\hline & $10(27,8)$ & $17(60,7)$ & {$[1,403-11,508]$} & 0,008 \\
\hline \multirow{2}{*}{$\begin{array}{l}\text { Patología de usuarios } \\
\text { (si/no) }\end{array}$} & $17(47,2)$ & $5(17,9)$ & 4,986 ** & \\
\hline & $19(52,8)$ & $23(82,1)$ & [1.347-18.450] & 0,0135 \\
\hline \multirow{2}{*}{$\begin{array}{l}\text { Flujograma de atención } \\
\qquad(s i / n o)\end{array}$} & $22(61,1)$ & $5(20,0)$ & $6,286 *$ & \\
\hline & $14(38,9)$ & $20(80,0)$ & {$[1,918-20,603]$} & 0,001 \\
\hline \multirow{2}{*}{$\begin{array}{l}\text { Entre } 0 \text { y } 4 \text { cambios } \\
\qquad \text { (si/no) }\end{array}$} & $10(27,8)$ & $16(57,1)$ & 0,398 ** & \\
\hline & $26(72,2)$ & $12(42,9)$ & {$[0,133-1,197]$} & 0,097 \\
\hline \multirow{2}{*}{$\begin{array}{l}\text { Entre } 5 \text { y } 9 \text { cambios } \\
\quad \text { (si/no) }\end{array}$} & $22(61,1)$ & $16(57,1)$ & 1,179 * & \\
\hline & $14(38,9)$ & $12(42,9)$ & {$[0,383-3,615]$} & 0,749 \\
\hline \multirow{2}{*}{$\begin{array}{l}\text { Entre } 10 \text { y } 14 \text { cambios } \\
\text { (si/no) }\end{array}$} & $6(16,7)$ & $5(17,9)$ & 0,756 ** & \\
\hline & $30(83,3)$ & $23(82,1)$ & {$[0,171-3,349]$} & 0,731 \\
\hline
\end{tabular}

$\mathrm{OR}^{*}=$ Odds Ratios crudos (Stress Laboral no modifica el efecto en más de un 10\%) $\mathrm{OR}^{* *}=$ Odds Ratios ajustado por Stress laboral (Prueba de Mantel-Haenszel de ${ }_{2}^{2}$, por presentar más de un 10\% de diferencia entre la OR cruda y la OR combinada con Stress Laboral).

IC $95 \%=$ Intervalo de confianza al 95\%.

La Tabla 3 señala que la calidad de insumos (OR crudo $=4,018 ; \mathrm{p}<$ $0,01)$, patologías de la atención (OR ajustado por estrés laboral = 4,986; $\mathrm{p}<0,05$ ) y flujograma de atención (OR crudo $=6,286 ; \mathrm{p}<$ $0,005)$ se asocian significativamente con los sintomas de ansiedad. Los profesionales que presentan entre 0 y 4 cambios (OR ajustado por estrés laboral $=0,398 ; p=0,097)$, entre 5 y 9 (OR crudo $=1,179 ; p$ $=0,749)$ y entre 10 y 14 cambios (OR ajustados por estrés laboral $=$ 0,$756 ; p=0,731$ ), no se asocian significativamente con sintomas de ansiedad.

\section{DISCUSIÓN}

El 27 de febrero de 2010, uno de los terremotos más intensos de la historia afectó la zona central de Chile, el cual provocó un tsunami que terminó por devastar las ciudades y localidades costeras. Ambos eventos catastróficos han tenido un efecto concreto no sólo en el medio ambiente físico y social de las personas, sino que también en sus cogniciones, emociones y comportamientos. ${ }^{20}$

Los hallazgos de este estudio, que apuntan al efecto en la salud mental de los profesionales de enfermería que sufrieron cambios en su ambiente laboral a consecuencia de la catástrofe, confirman dicha situación ya que presentaron síntomas de ansiedad, depresión y estrés laboral en diferentes niveles. Estos resultados concuerdan con la investigación de Jiménez y Cubillos ${ }^{28}$ donde los trabajadores presentaron un alto nivel de estrés postevento sísmico; con Gaborit en su investigación "Desastres y trauma psicológico", donde se identifican las distintas reacciones que tienen las personas en las diferentes fases después del evento traumático, entre las que se destacan reacciones secundarias tales como la depresión, la culpabilidad y la baja autoestima. ${ }^{19}$ La OPS confirma también estos resultados ya que postula que la catástrofe en sí, como todos sus efectos colaterales, generan frecuentemente trastornos de ansiedad y sintomas depresivos o ansiosos. ${ }^{21}$

Como lo expone Cohen R., en muchas situaciones los profesionales de la salud sobrevivientes son, al mismo tiempo, víctimas del evento y deben regresar a sus responsabilidades de emergencia y ayuda ${ }^{34}$, 
encontrándose, como en el caso de esta investigación, con recintos hospitalarios dañados estructuralmente a consecuencia del terremoto y un ambiente laboral muy distinto, organizacional como estructuralmente, al que estaban acostumbrados. Está ampliamente descrito en la literatura que en el ámbito de salud todo profesional de enfermería está expuesto a un grado de estrés y de ansiedad y sus consecuencias se revierten en una práctica profesional inadecuada a las personas que reciben sus cuidados ${ }^{10}$, lo que aumenta si se le suman factores como el tener que adaptarse a un ambiente laboral que presenta una serie de cambios, como lo postula Santos (2005). ${ }^{35}$ En diversas investigaciones se plantea que frente a la imposibilidad de responder o adaptarse rápidamente a un evento significativo e inesperado, como el terremoto y sus consecuencias, es probable que un ser humano continúe sintiéndose afectado durante varios meses con posterioridad al evento e, inclusive, manifieste signos de estrés, que se expresarían con diferente sintomatología, y en diversos ámbitos tales como el familiar, social y laboral. ${ }^{25}$

La lucha contra la ansiedad y el estrés, cualquiera sea su origen, constituye uno de los grandes empeños que deberán acometer tanto los gobiernos como las estructuras de dirección en los diferentes niveles. Las empresas que probablemente tengan más éxitos en el futuro serán las que ayuden a los trabajadores a hacer frente a la ansiedad y estrés y adapten las condiciones y la organización del trabajo a las actitudes humanas. Como se expuso, la profesión de enfermería es una de las ocupaciones más exigentes y estresantes de hoy en día, debido a la cantidad y diversidad de los factores de riesgo asociados con el ambiente de trabajo. Durante un día de trabajo típico, los enfermeros(as) pueden estar expuestos a una amplia gama de factores que generan estrés psicológico y ansiedad ${ }^{36}$, como lo fueron en esta investigación las modificaciones del ambiente laboral a raiz del sismo grado 8,8 en la escala de Richter, epicentro en la costa norte de la región del Bío-Bío. ${ }^{37}$

Como se expuso anteriormente, Chile tiene una larga experiencia en terremotos cuyas consecuencias afectan la vida de las personas, la infraestructura y los servicios básicos. ${ }^{34}$ Pero la restructuración de los establecimientos hospitalarios no sólo fue física sino que también logística, técnica y administrativa, generando cambios de diversa índole en el ambiente laboral destinados a hacer frente a la demanda en atención, los que han incidido directamente en la salud física y mental del profesional de enfermería involucrado, entre los cuales se destacan: la modificación del espacio de trabajo $(78,13 ; \mathrm{p}<0,001)$ que es además el cambio que mayor estrés produjo, y calidad de los insumos para la atención del usuario (57,81\%; $\mathrm{p}=0,0386)$. Es importante destacar que el 40\% de los sujetos en estudio indicaron que los cambios le perjudicaron; esto puede explicarse porque la antigüedad laboral que presentan los hace más propensos a la resistencia al cambio, ya que están habituados a un sistema y, por otro lado, como plantea Robbins S., puede deberse a la incertidumbre, preocupación, temor a lo desconocido, creencia de que el cambio no beneficia a la organización o bien por las pérdidas personales que esto acarrea ${ }^{38}$; es importante señalar que muchos profesionales de enfermería fueron cambiados de servicio e incluso de establecimiento. Algunas organizaciones tratan todos los cambios como algo que ocurre de manera accidental. Sin embargo, esto constituye un grave problema para encaminar la organización en un entorno tan cambiante. Las actividades de cambio en toda organización deben ser proactivas y con propó- sito final, o sea, que el cambio debe ser una actividad intencional y orientada a la meta, es decir, un cambio planeado. Los cambios repentinos son generadores de resistencia. ${ }^{38}$ Las resistencias al cambio no surgen necesariamente en forma estandarizada $\mathrm{y}$ pueden llevar a ansiedad y estrés en las personas que lo experimentan.

Por otro lado, se destaca que el cambio en la calidad de los insumos utilizados, patología de los usuarios y en el flujograma de atención se asocian estadísticamente con la presencia de ansiedad en los profesionales de enfermería ( $p<0,05)$, como lo explica Robbins, hacemos las cosas por hábitos, debemos tomar diariamente muchas decisiones, entonces para sobrellevar esta complejidad dependemos de hábitos o respuestas programadas. Pero cuando tenemos que hacer frente al cambio, esta tendencia a responder de la forma acostumbrada se convierte en una fuente de resistencia ${ }^{38}$ y justamente las tres variables (insumos, patologías de los usuarios y flujograma de atención) son las que mayor rutinización presentan en los establecimientos hospitalarios.

Los resultados de esta investigación reafirman lo descrito por autores como Collel y Pérez ${ }^{1,2}$, quienes indican que la presencia de estrés y ansiedad en el profesional de una institución de salud no siempre es de origen asistencial o bien por las exigencias del proceso productivo, el ambiente laboral es un factor evidentemente relacionado con la generación de sintomas de ansiedad, depresión y estrés; el ambiente laboral de las unidades de hospitalización es uno de los lugares de trabajo más estresante ${ }^{39}$, siendo la poca rotación, cambio de horario, objetivos poco claros, multitarea, demasiada responsabilidad sin incentivos, inseguridad laboral, ausencia de motivación para el crecimiento personal y cambios rápidos no compartidos o de las condiciones de trabajo o de responsabilidades algunos de los principales factores que los generan. Es evidente que los cambios ocasionados por el terremoto enfrentaron a los sujetos de estudio a condiciones nuevas a las que no estaban acostumbrados. Como lo expone en su estudio el Dr. Schnettler, el personal de salud queda situado tanto en el papel de víctima del desastre, como en el de auxiliador. El personal de salud ha manifestado sensaciones de frustración $\mathrm{y}$ cansancio por trabajar en situaciones estresantes, que se prolongarán a mediano plazo. ${ }^{18}$

A pesar que el terremoto fue una situación inesperada a la que se hizo frente con diferentes medidas de reestructuración logísticaadministrativa para absorber las demandas de la población, los cambios deben ser consensuados, en especial cuando permanecerán por algún tiempo, aunque sea sólo informando a los involucrados e incorporando sus experiencias y opiniones, ya que esto minimiza la resistencia a los mismos. Como esto ocurrió parcialmente, creemos que el profesional de enfermería se vio francamente afectado en su salud mental, por lo que se hace necesario tomar medidas preventivas realizando programas de intervención desde la perspectiva psicológica y, de este modo, contribuir a disminuir las manifestaciones de ansiedad y estrés, y así mejorar la calidad de vida en su trabajo. En todos los programas realizados algunos equipos multidisciplinarios de los servicios de salud con formación en salud mental que actuaron después del desastre ${ }^{20}$ fueron orientados a la comunidad, pero no al personal de salud. El comprender mejor cómo y a quiénes afecta psicológicamente un desastre socio-natural posibilita la intervención psicosocial de manera oportuna y efectiva ${ }^{19}$, teniendo en cuenta además que los desastres podrían aumentar en los próximos años, como consecuencia de los cambios climá- 
ticos, la sobrepoblación en zonas de riesgo y las acciones terroristas. Ello aumenta la importancia de desarrollar estrategias para mitigar sus consecuencias. Como lo afirman otras investigaciones mirando en perspectiva, el desastre del 27 de febrero de 2010 muestra la necesidad de acentuar la reflexión e investigación respecto de los desastres y sus implicaciones desde perspectivas psicosociales. $^{27}$

Es pertinente mencionar también que existen ciertas limitaciones de este estudio. En primer lugar, el universo fue limitado y debió extenderse no sólo a los profesionales de enfermería sino a todos los integrantes del equipo afectados por los cambios del ambiente laboral. Metodológicamente quizás hubiese sido más apropiado un análisis multivariado por medio de regresiones logísticas para haber establecido un modelo explicativo o predictivo de las varia- bles en estudio. Por tanto, para futuros estudios se considerará lo descrito anteriormente.

\section{AGRADECIMIENTOS:}

A Miguel Ángel López y Gonzalo Luengo Castañeda por sus aportes en el desarrollo de la investigación y artículo.

\section{Fuente de financiamiento}

Dirección de Investigación Universidad del Bío-Bío

*Presentado en Congreso de Salud Global: Santiago de Chile, enero 2013 (solo una parte de los resultados)

\section{REFERENCIAS}

1. Colell BR, Limonero GJ, Otero MD. Actitudes y emociones en estudiantes de enfermería ante la muerte y la enfermedad terminal. Investigación en Salud. 2003;(2):1-13.

2. Pérez $A C$, Alameda $C A$, Albeniz LC. La formación práctica en enfermeria en la escuela universitaria de enfermeria de la comunidad de Madrid. Opinión de alumnos y de los profesionales asistenciales. Un estudio cualitativo con un grupo de discusión. Rev Esp Salud Pública. 2002;76(5):517-530.

3. Blanco G. Estrés Laboral y Salud en las Enfermeras Instrumentistas. RFM(Caracas) [en línea]. 2004[citado 03 jun 2013];27(1):29-35. Disponible en: http://www.scielo.org.ve/scielo.php?script=sci_arttext\&pid=S0798$04692004000100006 \&$ Ing $=$ es.

4. Román HJ. Estrés y Burnout en profesionales de salud de los niveles primarios y secundarios de atención. Rev Cubana Salud Pública. 2003;29(2):103-10.

5. Faria $\mathrm{D}, \mathrm{Maia} \mathrm{M}$. Ansiedades y sentimientos de los profesionales de enfermeria en situaciones de terminalidad en oncología. Rev. Latino-Am. Enfermagem [en linea]. 2007 [citado 03 jun 2013];15(6):1131-1137. Disponible en: http://www. scielo.br/scielo.php?script=sci_arttext\&pid $=$ S0104-11692007000 600012\&Ing=en. http://dx.doi.org/10.1590/S0104-11692007000600012

6. Adriaenssens J, de Gucht V, Maes S. The Impact of traumatics events on emergency room nurses: Fidings from questionnaire survey. Int J Nurs Stud. 2012;49(11):1411-1422.

7. Tomás J, Maynegre $M$, Pérez $M$, Alsina $M$, Granell S. Sindrome de Burnout y riesgo suicida en enfermeras de atención primaria. Enferm Clin. 2010;20 (3):173-178.

8. Sanz SH, Caserio MA, Morante MA, Montero J, De La Cruz CR, Pallás A. Estudio de los niveles de ansiedad en los profesionales de una unidad neonatal. An Pediatr (Barcelona). 2012;77(1):22-27.

9. Flores $M E$, Troyo $R$, Valle MA, Vega MA. Ansiedad y estrés en la práctica del personal de enfermeria en un hospital de tercer nivel en Guadalajara. Revista electrónica de psicología Iztacala [en línea] 2010 [citado 8 jun 2013];13(1):1-17. Disponible en: http://www.iztacala.unam.mx/carreras/psicologia/psiclin/principal.html
10. Díaz $H$, Veliz $M$, Hernández $Y$. Evaluación de la ansiedad en profesionales del servicio a pacientes graves de un hospital universitario. Rev Cub Medic Intens Emerg. 2010;9(2) 1758-1763.

11. Sierra JC, Ortega V, Zubeidat I. Ansiedad, angustia y estrés: tres conceptos a diferenciar. Rev Mal-Estar Subj [on line]. 2003 [citado 8 jun 2013]; 3(1):10-59. Disponible en: http://pepsic.bvsalud.org/scielo.php?script=sci_arttext\&pid= S1518-61482003000100002\&Ing=pt\&nrm=iso

12. Ribera-Domene D, De la Peña E, Reig-Ferrer A, Romá-Ferri MT, Sanz I, Caruana-Vañó A. Estrés laboral y salud en profesionales de enfermeria. Estudio empírico en la provincia de Alicante. Alicante: Universidad de Alicante; 1993. $124 \mathrm{p}$.

13. Herrera $R$, Cassals $M$. Algunos factores influyentes en la calidad de vida laboral de enfermeria. Rev Cubana Enfermer [en línea]. 2005 [citado 8 jun 2013]; 21(1):1. Disponible en: http://scielo.sld.cu/scielo.php?script=sci_art text\&pid=S0864-03192005000100003\&lng=es

14. Trucco $M$, Valenzuela $P$, Trucco D. Estrés ocupacional en personal de salud. Rev Méd Chile [en línea]. 1999 [citado 8 jun 2013]; 127(12):1453-1461. Disponible en: http://www.scielo.cl/scielo.php?script=sci_arttext\&pid=S0034-9887199 9001200006\&lng=es\&nrm=iso

15. Hansen N, Sverke M, Naswall K. Predicting nurse burnout from demands and resources in three acute care hospitals under different forms of ownership: a cross-sectional questionnaire survey. Int J Nurs Stud [on line]. 2009 [cited 2013 Jun 10]: 46:96-107. Disponible en: http://cirrie.buffalo.edu/database/101543/

16. Glazer $S$, Gyurak A. Sources of oocupational stress among nurses in five countries. Int J Intercl Relat. 2008;32(1):49-66.

17. Decs; Descriptores de ciencias de la salud [on line]. Sao Paulo: BIREMEOPS-OMS; 2013[citado 8 jun 2013]. Disponible en: http://decs.bvs.br/E/homepagee.htm

18. Schenettler D. Efectos del megasismo del 27 de febrero de 2010 en Hospital regional de Talca. Análisis bioético enfocado en la prevención. Rev Chil Salud Pública. 2013;17(1):28-34. 


\section{REFERENCIAS}

19. Gaborit M. Desastres y Trauma psicológico. Pensamiento Psicológico. 2006;2(7): 15-39.

20. Sigales S. Catástrofe, víctimas y trastornos: Hacia una definición en psicología. Anales de Psicología. 2006;22(1):11-21.

21. Leiva M, Quintana G. Factores Ambientales y Psicosociales Vinculados a Sintomas de Ataque de Pánico Después del Terremoto y Tsunami del 27 de Febrero de 2010 en la Zona Central de Chile. Ter psicol [en línea]. 2010 [citado 28 ene 2014];28(2):161-167. Disponible en: http://www.scielo.cl/ scielo.php?script=sci_arttext\&pid=S0718-48082010000200004\& lng=es\&tlng=es. 10.4067/S0718-48082010000200004

22. López E, Santana P. El terremoto de 2010 en Chile: respuesta del sistema de salud y de la cooperación internacional. Rev Panam Salud Pública. 2011;30(2)160-166.

23. Reyes H. Chile se pone de pie otra vez. Rev Méd Chile [en línea]. 2010 [citado 30 ene 2014];138(3):267-269. Disponible en: http://www.scielo.cl/ scielo.php?script=sci_arttext\&pid=S0034-98872010000300001\&Ing=es. http://dx.doi.org/10.4067/S0034-98872010000300001

24. Souto $A$, Talavera M, Moruno P. Los desastres naturales desde la perspectiva ocupacional. TOG (A Coruña) [en línea]. 2013 [citado 30 ene 2014];10(Supl 8):75-80. Disponible en: www.revistatog.com/suple/num8/ desastres.pdf

25. Loubat M, Fernández AM, Morales M. La Experiencia de Peralillo: Una Intervención Psicológica para el Estado de Emergencia. Ter psicol [en línea]. 2010[citado 30 ene 2014];28(2):203-207. Disponible en: http://www.scielo. $\mathrm{cl} /$ scielo.php?script=sci_arttext\&pid=S0718-48082010000200009\&Ing= es\&tlng=es. 10.4067/S0718-48082010000200009

26. Huggard P. Caring for the Carers: the emotional effects of disasters on health care professionals. Australas J Disaster Trauma Stud. 2011;2.

27. Figueroa R, Marin H, Gonzalez M. Apoyo psicológico en desastres: Propuesta de un modelo de atención basado en revisiones sistemáticas y metaanálisis. Rev méd Chile [en línea]. 2010[citado 3 ene 2014]; 138(2):143-151. Disponible en: http://www.scielo.cl/scielo.php?script=sci_arttext\&pid=S00 34-98872010000200001\&

28. Jiménez $A$, Cubillos $R$. Estrés percibido y satisfacción laboral después del terre- moto ocurrido el 27 de Febrero de 2010 en la Zona Centro-Sur de Chile. Ter Psicol. 2010; 28(2):187-192.

29. Cova F, Rincón P. El terremoto y tsunami del $27-F$ y sus efectos en la salud mental. Ter Psicol [en línea]. 2010 [consultado 30 ene 2014];28(2):179-185. Disponible en: http://www.redalyc.org/articulo.oa?id=78516731006\&idp=1\& cid $=1077666$

30. Romo M, Rodriguez N. Traza tu rumbo. Madrid: Lid; 2010.

31. Cutshall S, Wentworth L, Wahner-Roedler D, Vincent A, Schmidt J, Loehrer L, Stephen S, Brent A. Evaluation of Biofeedback-Assisted Meditation Program as a Stress Management Tool for Hospital Nurses: A Pilot Study. EXPLORE: J Sci Healing. 2011; 7(2):110-112.

32. Jélvez $C$, lbañez J P, Olivares V. Validez factorial del Maslach Burnout Inventory Human Services (MBI-HSS) en profesionales chilenos. Cienc y trab. 2011;13 [41]: 176-180. Citado 10 mayo 2013. Disponible: http://edu.uamericas.cl/ psicologia/files/2011/11/articulo-carolina-jelvez.pdf

33. Montón C, Pérez Echeverría MI, Campos R y cols. Escalas de ansiedad y depresión de Goldberg: una guia de entrevista eficaz para la detección del malestar psiquico. Aten Primaria. 1993; 12(6):345-9.

34. Cohen R. Lecciones aprendidas durante desastres naturales: 1970-2007. Rev Perú Med Exp Salud Pública. 2008; 25(1):109-117.

35. Santos JA. Manual: PRANES. El Salvador: Acción Consultores; 2005.

36. Blanco G. Estrés Laboral y Salud en las Enfermeras Instrumentistas. RFM [en línea]. 2004 [consultado 10 jun 2013];27(1):29-35. Disponible en: http://www. scielo.org.ve/scielo.php?script=sci_arttext\&pid=S0798-04692004000 $100006 \&$ Ing=es

37. U.S. Department of the Interior. Earthquake Hazards Program: 2010 February 27 06:34:14 UTC. [on line]. Virginia: U.S. Geological Survey; 2010. [cited February 06 2013]. Disponible en: http://earthquake.usgs.gov/earthquakes/ eqinthenews/2010/us2010tfan/.

38. Robbins S, Coulter M. Manejo del cambio y la innovación. Cap. XIII. En: Administración. $8^{a}$ ed. México: Pearson Educación-Prentice Hall; 2008. p. 313-323.

39. Marriner Tomey A. Gestión y Dirección de Enfermeria. Madrid: Elsevier- Mosby; 2006. p. 28-50. 